\title{
Attitude stabilization of slosh-coupled satellite with flexible appendage
}

\author{
J. E Benmansour ${ }^{(1)^{*}}$, b. Khouane ${ }^{(2)}$ \\ ${ }^{(1)}$ Département de Recherche en Mécanique Spatiale Centre de Développement des Satellites \\ (CDS) Oran, Algeria \\ ${ }^{(2)}$ Département de Mission et Systèmes Spatiaux ; Centre de Développement des Satellites (CDS) \\ Oran, Algeria \\ ¡jebenmansour@cds.asal.dz
}

\begin{abstract}
In this paper, a robust control method for attitude stabilization flexible satellite and propellant slosh torques suppression is proposed based on the combination of a composite disturbance observer (CDO) and feed-forward controller. First, a CDO is constructed to estimate the lumped disturbances, including, the external disturbing forces where the lumped disturbances in the system are equivalent into two parts: a slow varying disturbance and periodic disturbance. Then a PD controller is adopted to stabilize the system and restrain the residue disturbance. Finally, the simulation results for flexible satellite verify that the proposed method can make the satellite asymptotically stable and demonstrate the effectiveness of the proposed scheme.
\end{abstract}

Keywords: composite disturbance observer, flexible satellite, attitude control, slosh, coupling dynamics.

\section{INTRODUCTION}

The attitude control and stability of flexible satellites interacting with fluid motion have become an increasingly important task in space missions due to the structural oscillations induced by flexible appendages including the effect of sloshing.

In the past years, the attitude control for satellite was based on a rigid body taking into account only the external torque disturbances $[1,2]$. However, several sources of flexibilities resulting from the conception of the new communication satellites such as the solar arrays and antennas affect the complying with the mission requirements. These coupling torques between the rigid-body and flexible modes can significantly pose a structural problem to the attitude stabilization and the performance of the system [3,4]. Hence, various control techniques have been developed to deal with system's disturbances to get a fast response and high control precision.

Proportional-Integral-Derivative approaches are the most widely and major linear control algorithm used in the field of satellite attitude systems to improve system convergence under Earth gravity [5,6]. However, the control structure has limitations to reject the multiple disturbances.

The sliding mode control (SMC) was applied to the satellite attitude control system due to its strong robustness against disturbances [79] but in SMC the chattering phenomenon that results from discontinuous control presents a severe problem and the mentioned methods ignored the flexible modes and only dealt with a single equivalent disturbance.

To the same goal, the $\mathrm{H}^{\infty}$ control and linear matrix inequality (LMI) are used to achieve the required objective of the satellite attitude control system in the presence of disturbance. The complexity of these methods requires the use of actuators that consume more energy. Another interesting work, which employed the linear quadratic regulator (LQR) and linear quadratic Gaussian (LQG) for studying the motion of liquid propellants in the satellite in which the sloshing phenomenon is represented by its mechanical system of pendulum-type $[10,11]$. However, these methods have their particular characteristics in that all the states have to be known.

As effective disturbance attenuation, the development of disturbance observer techniques will be a good way to alleviate the restrictions. Therefore, this approach has attracted the attention of many researchers, since it has less conservativeness for several forms of disturbances and would be easy to integrate with feedback controllers such as: $\mathrm{PD}, \mathrm{H} \infty$, and variable structure controllers [12-15]. 
In this study, we investigate the control design scheme for attitude stabilization of slosh-coupled satellite with flexible appendage. Considering the internal disturbances (the structural vibration and liquid sloshing) and the external disturbances acting on the satellite, the composite attitude controller scheme is designed to perform good disturbance attenuation ability and suppress the liquid sloshing and the vibration of the flexible appendages for pitch attitude stabilization

The rest of the paper is structured as follows. Section 2 introduces the mathematical equations of the coupled system, which included the equations of the flexible satellite and coupling dynamics of liquid propellant. The composite disturbance observer is proposed in section 3 . Section 4 gives the detailed of composite attitude controller design. The results of the numerical simulations are discussed and analysed in Section 5. Finally, Section 6 concludes the paper by giving a brief summary and discuss the future work.

\section{MODEL FORMULATION}

The satellite is modelled as a main rigid body with flexible appendages and propellant tanks, which generates the liquid sloshing, (as shown in Fig 1).

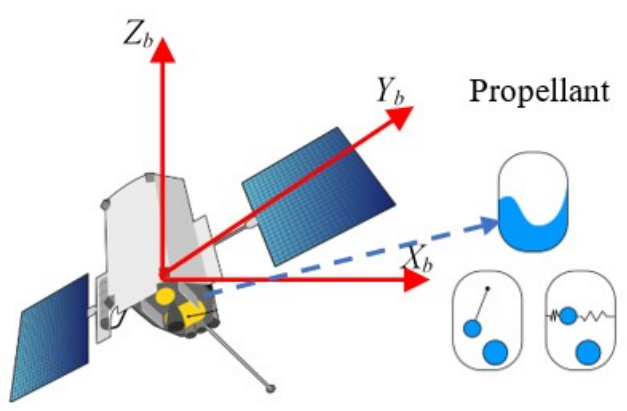

Fig 1Flexible satellite with movement of the propellant

In order to simplify the model, only singleaxis rotation is considered, similarly, to [8], the pitch axis model can be described from the nonlinear attitude dynamics of the rigid spacecraft with flexible appendages and sloshing mode as follows:

$$
\begin{aligned}
& J \ddot{\theta}+F_{1} \ddot{\eta}+F_{2} \ddot{\sigma}=u_{y}+w(1) \\
& \left\{\begin{array}{l}
\ddot{\eta}+2 \xi_{\eta} \omega_{\eta} \dot{\eta}+\omega_{\eta}^{2} \eta+F_{1}^{T} \ddot{\theta}=0 \\
\ddot{\sigma}+2 \xi_{\sigma} \omega_{\sigma} \dot{\sigma}+\omega_{\sigma}^{2} \sigma+F_{2}^{T} \ddot{\theta}=0
\end{array}\right.
\end{aligned}
$$

where $\theta, J, F_{1,2}, u_{y}, w$ are the attitude angle, the moment of inertia of the satellite, the rigid elastic coupling matrix, the control torque, the merged disturbances, respectively; $\eta, \sigma$ represents the flexible, and the sloshing model coordinate, while $\xi_{\eta, \sigma}$ are the damping ratio and $\omega_{\eta, \sigma}$ are the model frequency.

Combining (1) with (2), it can be obtained as:

$$
\begin{aligned}
\left(J-F_{1} F_{1}^{T}-F_{2} F_{2}^{T}\right) \ddot{\theta} & =F_{1}\left(2 \xi_{\eta} \omega_{\eta} \dot{\eta}+\omega_{\eta}^{2} \eta\right) \\
& +F_{2}\left(2 \xi_{\sigma} \omega_{\sigma} \dot{\sigma}+\omega_{\sigma}^{2} \sigma\right) \\
& +w+u_{y}
\end{aligned}
$$

where

$F_{1}\left(2 \xi_{\eta} \omega_{\eta} \dot{\eta}+\omega_{\eta}^{2} \eta\right)+F_{2}\left(2 \xi_{\sigma} \omega_{\sigma} \dot{\sigma}+\omega_{\sigma}^{2} \sigma\right)+w$

is considered as the total disturbance caused by elastic vibration of the flexible appendages, the sloshing mode, and the external environment.

Denote $x_{1}=\theta$ and $x_{2}=\dot{\theta}, \quad X=\left(x_{1}, x_{2}\right)^{T}$, then (3) can be transformed into

$\left\{\begin{array}{l}\dot{x}_{1}=x_{2} \\ \dot{x}_{2}=b u_{y}+b d_{y}\end{array}\right.$

where

$$
\begin{aligned}
b= & \left(J-F_{1} F_{1}^{T}-F_{2} F_{2}^{T}\right)^{-1} \text { and } \\
d_{y} & =F_{1}\left(2 \xi_{\eta} \omega_{\eta} \dot{\eta}+\omega_{\eta}^{2} \eta\right) \\
& +F_{2}\left(2 \xi_{\sigma} \omega_{\sigma} \dot{\sigma}+\omega_{\sigma}^{2} \sigma\right)+w
\end{aligned}
$$

are the disturbances which represent the internal (flexible appendages, sloshing mode) and the external disturbances (solar pressure).

Then, Eq. (3) can be simplified as

$\left(J-F_{1} F_{1}^{T}-F_{2} F_{2}^{T}\right) \ddot{\theta}=u_{y}+d_{y}$

Hence Eq. (1) can be rewritten into the following form:

$$
\dot{X}=A X+B\left(u_{y}+d_{y}\right)
$$

where

$$
A=\left[\begin{array}{ll}
0 & 1 \\
0 & 0
\end{array}\right], B=\left[\begin{array}{c}
0 \\
b=\left(J-F_{1} F_{1}^{T}-F_{2} F_{2}^{T}\right)^{-1}
\end{array}\right]
$$

\section{COMPOSITE DISTURBANCE OBSERVER}

The disturbance can be considered as two parts [16,17]; nonlinear slow varying disturbance which can be used to describe 
the vibration of flexible appendages, and the periodic disturbance generated by the unmodelled dynamics and external disturbances.

To design the composite disturbance observer for the pitch axis, the following assumptions are needed:

1. Assume $d_{y}=d_{y 1}+d_{y 2}$, where $d_{y}$ is the equivalent disturbance which includes the vibration, external perturbations, and unmodelled dynamics.

2. Assume $d_{y 1}$ varies very slowly, i.e., $\dot{d}_{y 1} \approx 0$.

3. Assume $d_{y 2}$ is the periodic disturbance with angular frequency $\Omega$, and $\ddot{d}_{y 2}+\Omega^{2} d_{y 2}=0$.

Let $\xi_{y 1}=d_{y 1}, \xi_{y 2}=d_{y 2}, \xi_{y 3}=\dot{d}_{y 2}$ and $\xi_{y}=\left(\xi_{y 1}, \xi_{y 2}, \xi_{y 3}\right)^{T}$, then the disturbance model can be derived from the above assumptions as:

$$
\begin{aligned}
& \dot{\xi}_{y}=M_{y} \xi_{y} \\
& d_{y}=\xi_{y 1}+\xi_{y 2}=C_{y} \xi
\end{aligned}
$$

where

$$
M_{y}=\left[\begin{array}{ccc}
0 & 0 & 0 \\
0 & 0 & 1 \\
0 & -\Omega^{2} & 0
\end{array}\right], C_{y}=\left[\begin{array}{lll}
1 & 1 & 0
\end{array}\right] .
$$

According to Eq. (6), (7), and Eq. (8), the pitch composite disturbance observer can be designed as:

$$
\left\{\begin{array}{l}
\hat{\xi}=p+L J \dot{\theta} \\
\dot{p}=\left(M_{y}-L C_{y}\right)(p+L J \dot{\theta})-L u_{y}
\end{array}\right.
$$

where

$p$ is the internal variable vector of the observer and $L$ is the observer gain matrix.

The performance of the pitch composite disturbance observer is analyzed as follows. According to Eq. (9) and Eq. (5), the following equation can be derived as:

$$
\begin{aligned}
\dot{\hat{\xi}}_{y} & =\left(M_{y}-L C_{y}\right) \hat{\xi}_{y}+L\left(\ddot{\theta} J-u_{y}\right) \\
& =\left(M_{y}-L C_{y}\right) \hat{\xi}_{y}+L d_{y} \\
& =M_{y} \hat{\xi}_{y}+L d_{y}-L \hat{d}_{y} \\
& =M_{y} \hat{\xi}_{y}+L\left(d_{y}-\hat{d}_{y}\right)
\end{aligned}
$$

The disturbance estimation error is designed as

$\tilde{d}_{y}=d_{y}-\hat{d}_{y}$

The error dynamics of the composite disturbance observer can be defined as

$\tilde{\xi}_{y}=\xi_{y}-\hat{\xi}_{y}$

Combining Eq. (10) and Eq. (12), the error dynamic of the observer can be derived as:

$\dot{\tilde{\xi}}_{y}=M_{y} \tilde{\xi}_{y}-L C_{y} \tilde{\xi}_{y}=\left(M_{y}-L C_{y}\right) \tilde{\xi}_{y}$

The stability of the closed-loop system under the composite controlleris analysed as follow:

Based on Eq. (13), Since $\left(M_{y}-L C_{y}\right)$ can be Hurwitz by choosing suitable $L$, all the eigenvalues of $\left(M_{y}-L C_{y}\right)$ have negative real parts.

Let $M_{0}=M_{y}-L C_{y}$, there exists

$P_{y}>0, \mathrm{Q}_{y}>0$ such that:

$P_{y} M_{0}+M_{0}^{\mathrm{T}} P_{y}=-Q_{y}$

Choose Lyapunov function as:

$V_{1}=\tilde{\xi}_{y}^{T} P_{y} \tilde{\xi}_{y}$

Then the time derivative of Eq. (15) along Eq. (13) and Eq. (14) can be obtained as:

$$
\begin{aligned}
\dot{V}_{1} & =\dot{\tilde{\xi}}_{y}{ }^{T} P_{y} \tilde{\xi}_{y}+\tilde{\xi}_{y}{ }^{T} P_{y} \dot{\tilde{\xi}}_{y} \\
& =-\tilde{\xi}_{y}{ }^{T} Q_{y} \tilde{\xi}_{y}<0
\end{aligned}
$$

theory of Lyapunov stability, it can be concluded that the error dynamics is stable asymptotically.

\section{COMPOSITE ATTITUDE CONTROLLER DESIGN}

In this study, the composite control law for system (6) is constructed as follow:

$u_{y}=-K X-\hat{d}_{y}$

where $K$ is the feedback control law to be designed. 
The block diagram of the composite controller is giveninFig 2 .

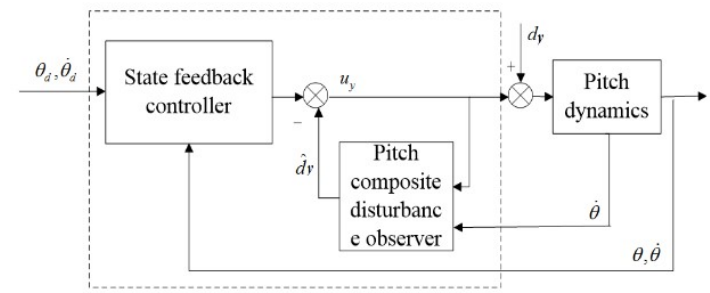

Fig 2: Block diagram of the composite controller

Combining Eq. (6) with Eq. (17) the closedloop system is governed by:

$$
\dot{X}=(A-B K) X+B \tilde{d}_{y}(18)
$$

Let $\bar{A}=A-B K$ and $\tilde{d}_{y}=C_{y} \tilde{\xi}_{y}$, then:

$$
\dot{X}=\bar{A} X+B C_{y} \tilde{\xi}_{y}(19)
$$

Since $(A, \mathrm{~B})$ is controllable, $\bar{A}$ can be Hurwitz by choosing suitable $K$, there must exist $P, Q>0$ such that:

$$
P \bar{A}+\bar{A}^{T} P=-Q
$$

Choose Lyapunov function as:

$$
V_{2}=X^{T} P X+\tilde{\xi}_{y}{ }^{T} P_{y} \tilde{\xi}_{y}
$$

Differentiating Eq. (21) along Eq. (16), Eq. (18) and Eq. (20) gives:

$$
\begin{aligned}
\dot{V}_{2} & =\dot{X}^{T} P X+X^{T} P \dot{X}+\dot{\tilde{\xi}}_{y}{ }^{T} P_{y} \tilde{\xi}_{y}+\tilde{\xi}_{y}{ }^{T} P_{y} \dot{\tilde{\xi}}_{y} \\
& =-X^{T} Q X+2 X^{T} P B C_{y} \tilde{\xi}_{y}-\tilde{\xi}_{y}{ }^{T} Q_{y} \tilde{\xi}_{y} \\
& \leq-\lambda_{\min }(Q)\|X\|^{2}+2\|P\|\left\|C_{y}\right\|\|X\|\left\|\xi_{y}\right\| \\
& -\lambda_{\min }\left(Q_{y}\right)\left\|\tilde{\xi}_{y}\right\|^{2}
\end{aligned}
$$

Let

$$
\lambda_{1}=\lambda_{\min }(Q), \lambda_{2}=\max \left(\|P\|\left\|C_{y}\right\|\right),
$$

$\lambda_{3}=\lambda_{\min }\left(Q_{y}\right)$, then Eq. (22) can be rewritten as:

$$
\begin{aligned}
\dot{V}_{2} & \leq-\lambda_{1}\|X\|^{2}+2 \lambda_{2}\|X\|\left\|\tilde{\xi}_{y}\right\|-\lambda_{3}\left\|\tilde{\xi}_{y}\right\|^{2} \\
& =-\left(\lambda_{1}-\frac{\lambda_{2}^{2}}{\sqrt{\lambda_{3}}}\right)\|X\|^{2}-\left(\frac{\lambda_{2}}{\sqrt{\lambda_{3}}}\|X\|-\sqrt{\lambda_{3}}\left\|\tilde{\xi}_{y}\right\|\right)^{2}
\end{aligned}
$$

$$
\text { If } \lambda_{1}-\frac{\lambda_{2}^{2}}{\sqrt{\lambda_{3}}}>0, \quad \dot{V}_{2} \leq 0 . \quad \text { According }
$$

toLyapunov stability theory, it can be concluded that the state and the disturbance estimation error can converge to the origin asymptotically as $t \rightarrow \infty$.

\section{SIMULATION RESULTS}

In this section, the numerical simulation will demonstrate the efficiency of the proposed composite controller, where single-axis spacecraft attitude control has been considered, the same simulation conditions in [11] are used by adding the space external environmental disturbance acting on the satellite, which described as $d_{y 2}=\sin \left(\frac{\pi}{2} t\right)$ N.m.

The gain of disturbance observer is chosen as $L=[15,1.15,5]^{T}$ and the PD control gain is obtained as $K=\left[\begin{array}{ll}245 & 1550\end{array}\right]$.

The total disturbance $d_{y}$, which represents the vibrations resulting from the flexible appendages, sloshing mode, and external disturbance is illustrated in Fig.3. It can be shown that the curve of observer and the real disturbances almost overlap. Thus it can be conclude that the global disturbances can be effectively estimated by composite disturbance observer, Moreover, Figs 4 and 5 describe two types of disturbance $d_{y 1}$ and $d_{y 2}$, where the slow varying disturbance $d_{y 1}$ consists of the elastic vibration with fuel sloshing, and $d_{y 2}$ is the periodic disturbance which includes the external environmental disturbance. In the both figures, the disturbances are estimated synchronously where the errors have been reduced to the lowest.

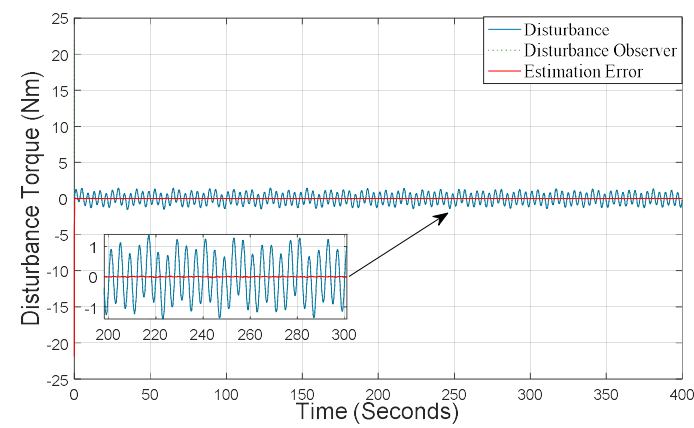

Fig 3: Time response of disturbance dy and estimation 


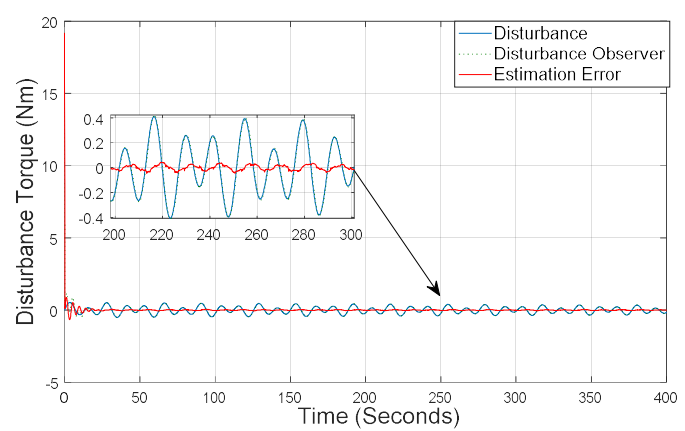

Fig 4: Time response of disturbance dy1 and estimation

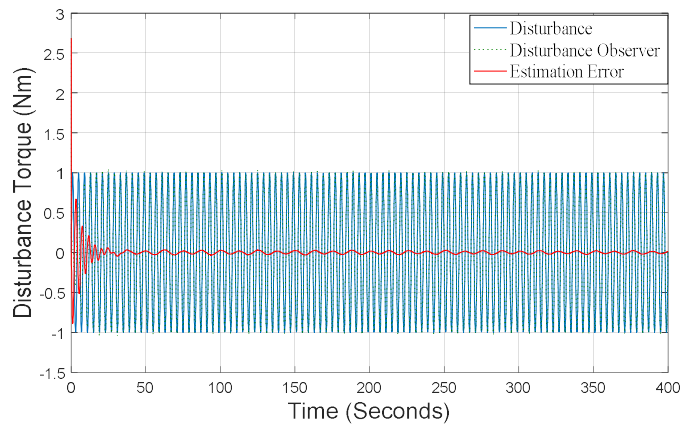

Fig 5: Time response of disturbance dy2 and estimation

Figures 6 and 7 demonstrate time histories of the attitude angle and angle velocity respectively. As we can see, the attitude control accuracy and attitude stabilization have better dynamic response performance controlled by the composite controller compared to the existing control algorithm (PD controller). We can come to a conclusion that the attitude angle and attitude angular rate are more precise and more stable compared with PD controller.

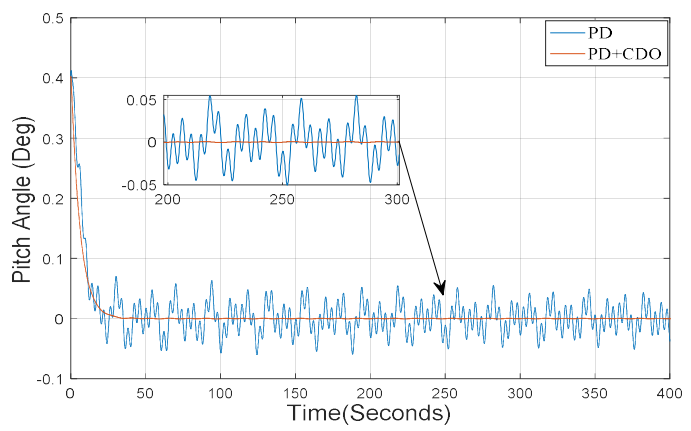

Fig 6: Time response of pitch attitude angle

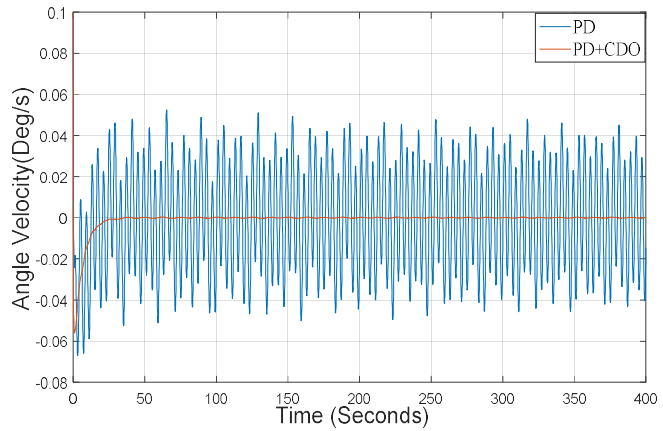

Fig 7: Time response of pitch attitude velocity

For more detailed analysis, the root mean square (RMS) values of error results are computed for the time period $200-400 \mathrm{sec}$, which are presented in the following Table 1

Table 1: RMS errors of the attitude

\begin{tabular}{|l|c|c|}
\hline & $\begin{array}{c}\text { RMSof } \\
\text { attitude } \\
\text { (deg) }\end{array}$ & $\begin{array}{c}\text { RMS of } \\
\text { attitude rate } \\
\text { (deg/s) }\end{array}$ \\
\hline PD Controller & Pitch 0.033 & $\begin{array}{c}\text { Pitch } \\
0.026\end{array}$ \\
\hline $\begin{array}{l}\text { PD+CDO } \\
\text { controller }\end{array}$ & $\begin{array}{c}\text { Pitch } \\
\text { Pitch } \\
8.0057\end{array}$ & $8.732 \mathrm{e}-04$ \\
\hline
\end{tabular}

\section{Monte Carlo Simulation}

To evaluate the performance of the proposed controller, a series of Monte Carlo simulations have been carried out as shown in Fig 8. The tests consist of 10000 simulation runs. The aim is to evaluate the accuracy performance of the method used during attitude control. Indeed, for each Monte-Carlo run, the Euler angles control errors and angle rates control errors converge; in addition, the global magnitude error does not exceed 0.055 (deg), for every simulation in attitude angle errors and $9.10^{-3}$ (deg/s) in attitude velocity errors, it can be confirmed that the performance of the proposed controller have been reached against various disturbances. 

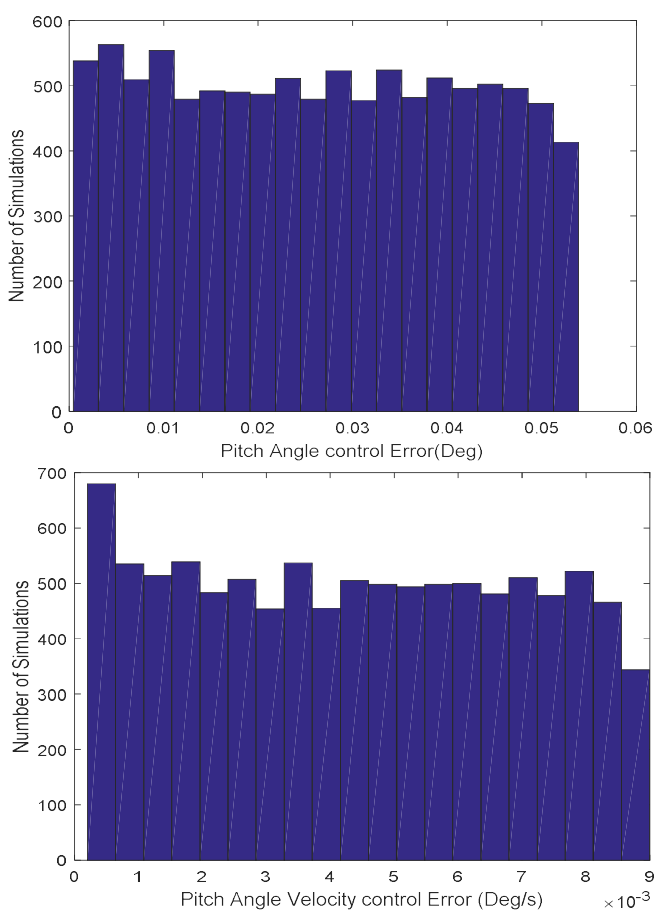

Fig. 8: Histogram of Pitch angles and velocity control errors using CDO+PD Controller

\section{CONCLUSIONS}

The robust control method for attitude stabilization of slosh-coupled satellite with flexible appendage is studied in this paper. The dynamic model for a satellite with multiple flexible modes is firstly proposed and presented. A new algorithm method based on composite disturbance observer with PD controller is designed to achieve and guarantee successful estimation. Further, the robust stability of the system in the presence of disturbances is proved. Noted that the advantages of this approach are can both estimate slowly-varying disturbances and other objects with multiple disturbances.

\section{Acknowledgements}

The authors wish to thanks the head Department of Research in Space Mechanics, satellite development centre (CDS) in Oran.

\section{References}

[1] H. Bang, J.S.Lee andY.J.Eun, "Nonlinear attitude control of a rigid spacecraft by feedback linearization", J. Mech. Sci. Technol., Vol.18, No.2, 2004, pp.203-210.

[2] Z.Chen,J. Huang, "Attitude tracking and disturbance rejection of rigid spacecraft by adaptive control", IEEE Trans. Autom. Control., Vol.54, No.3, 2009, pp.600-605.

[3] C. Catherine. "Hinf Controller Design and mAnalysis: Powerful Tools for Flexible Satellite Attitude Control". In : AIAA Guidance, Navigation, and Control Conference. 2010. p. 7907.

[4] C. G Mayhew, G. R. Sanfelice, and A. R.
Teel. "Robust global asymptotic attitude stabilization of a rigid body by quaternionbased hybrid feedback." In Proc., 48h IEEE Conf. on Decision and Control (CDC) Held Jointly with 2009 28th Chinese Control Conf., 2522-2527. New York: IEEE

[5] B. Scott. "Comparison of PD and LQR methods for Spacecraft attitude control using star trackers," IEEE Transactions on World Automation Congress, 2006, pp. $1-6$.

[6] Y. Li, Z.Sun, and D. Ye,. "Robust linear PID controller for satellite attitude stabilization and attitude tracking control", Int. J. Space Science and Engineering, Vol. 4, No. 1, 2016, pp.64-75.

[7] P.M. TIWARI, S. JANARDHANAN, and $U$ NABI. "Attitude control using higher order sliding mode". Aerospace Science and Technology, 2016, vol. 54, p. 108-113.

[8] B. Hyochoong, HA, Cheol-Keun, and K Jin Hyoung. "Flexible spacecraft attitude maneuver by application of sliding mode control". Acta Astronautica, 2005, vol. 57, no 11, p. 841-850.

[9] M.Reyhanoglu, and J. R. Hervas, "Nonlinear control of a spacecraft with multiple fuel slosh modes," Decision and Control and European Control Conference (CDC-ECC), 2011 50th IEEE Conference on, IEEE, 2011, pp. 61926197.

[10] Y. Li, D. Ye,"Time efficient sliding mode controller based on Bang-Bang logic for satellite attitude control” "Aerosp.Sci.Technol. 75,2018, pp.342-352.

[11] DE SOUZA, Luiz Carlos Gadelha et DE SOUZA, Alain G. Satellite attitude control system design considering the fuel slosh dynamics. Shock and Vibration, 2014, vol. 2014..

[12] J. E . Benmansour, M. S. Mohammed, and A. Bellar. Extended state observer based control of attitude stabilization for flexible spacecraft with solar pressure and slosh disturbances. In 2017 5th International Conference on Electrical Engineering-Boumerdes, IEEE (ICEE-B) , 2017, October ,pp. 1-6.

[13] J .E. Benmansour, Z. Wu, et al . "Disturbance Observer based on Controller for Roll/Yaw Attitude Stabilization of Flexible Spacecraft". Electrotehnica, Electronica, Automatica, 64(2), 157.

[14] X. Wei, L .Guo . "Composite disturbance-observer-based control and $\mathrm{H}_{\infty}$ control for complex continuous models". International Journal of Robust and Nonlinear Control, 20(1), 2016, 106-118.

[15] J. E. Benmansour, Z . Wu, and B .Khouane,. "Disturbance Observer based on Controller for Roll/Yaw Attitude Stabilization of Flexible Spacecraft". Electrotehnica, Electronica, Automatica,2016. 64(2)

[16] Z . Wang , Z Wu, L. Li, and L. Yuan. " A composite anti-disturbance control scheme for attitude stabilization and vibration suppression of flexible spacecrafts".Journal of Vibration and Control,23(15), 2017, 24702477.

[17] J. E . Benmansour, B. Khouane. "Active Control Design Approach for Roll/Yaw Attitude Satellite Stabilization with Flexible Vibration”. Aut. Control Comp. Sci. 54, 2020, 70-79. 